\title{
An unusual case of metastasis of a pulmonary undifferentiated pleomorphic sarcoma to the right ventricle: a case report
}

\author{
Guodong Xu*, Xinbao Shi and Guofeng Shao
}

\begin{abstract}
Introduction: Undifferentiated pleomorphic sarcoma is defined as a pleomorphic high-grade sarcoma whose line of differentiation cannot be determined. These tumors constitute less than $5 \%$ of all sarcomas in adults. Cardiac neoplasms are rare, and most are metastatic in origin. More than one-third of cardiac metastases originate from lung cancer. Symptoms of cardiac neoplasms usually appear late in the course of the disease and are often ignored because of the more severe effects of the primary malignancy or its therapy. We present the case of a patient with undifferentiated pleomorphic sarcoma of the lung presenting with symptomatic right-heart failure secondary to cardiac metastasis. The purpose of this report is to present this unusual case.

Case presentation: Our patient was a 59-year-old Chinese woman with symptomatic metastasis of an undifferentiated pleomorphic sarcoma of the lung to the right ventricle. She had a history of a stage IV, pulmonary, undifferentiated pleomorphic sarcoma that had been successfully treated with chemotherapy and radiotherapy 4 years ago. A complete response was obtained, and she was in remission until the cardiac metastasis. She underwent surgical excision of the cardiac mass because it caused dyspnea and posed a high risk of sudden death, pulmonary embolism or tricuspid obstruction. Histopathological and immunohistochemical examinations of the surgical specimen established the diagnosis of undifferentiated pleomorphic sarcoma and confirmed that the cardiac tumor was a metastasis from the lung.

Conclusions: In patients who have known metastatic neoplasms and present with cardiac manifestations, whether detected during history taking or physical examination, the clinician should be alert to the possibility of cardiac metastases. In patients with cardiac metastases, the therapeutic alternatives are limited to palliative treatment of symptoms and chemotherapy. In some patients, surgery can be used to relieve symptoms. We have reported the first case of symptomatic cardiac metastases from an undifferentiated pleomorphic sarcoma of the lung. Our patient underwent surgical resection, and her symptoms improved significantly. This case is unique because it is the only reported case of undifferentiated pleomorphic sarcoma of the lung which metastasized to the heart, and in which symptomatic improvement was effectively obtained with surgical resection.
\end{abstract}

Keywords: Cardiac metastasis, Lung undifferentiated pleomorphic sarcoma, Surgical resection

\section{Introduction}

Undifferentiated pleomorphic sarcoma is defined as a pleomorphic high-grade sarcoma whose line of differentiation cannot be determined. These tumors constitute less than $5 \%$ of all sarcomas in adults [1]. From a clinical viewpoint, undifferentiated pleomorphic sarcomas are deeply located

\footnotetext{
*Correspondence: xuguodong5306750@gmail.com

Department of Thoracic \& Cardiovascular Surgery, Lihuili Hospital, Ningbo Medical Center, Affiliated Hospital of Medical School of Ningbo University, Xingning Road, Ningbo 315041, P. R. China
}

tumors that show progressive and rapid growth. The mean 5 -year survival rate ranges from $50 \%$ to $60 \%$ [2].

Cardiac neoplasms are rare, and most are metastatic in origin. Cardiac metastases are found at autopsy in 6\% to $20 \%$ of patients with malignant neoplasms [3]. More than one-third $(36 \%)$ of cardiac metastases originate from lung cancer. Non-solid primary malignancies such as leukemia, lymphoma and Kaposi sarcoma account for $20 \%$ of cardiac metastases, breast carcinoma for $7 \%$ and esophageal carcinoma for $6 \%$ [4].

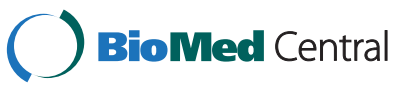


Symptoms of cardiac neoplasms usually appear late in the course of the disease and are often ignored because of the more severe effects of the primary malignant disorder or its therapy. Consequently, cardiac neoplasms, especially metastatic ones, are often not discovered until autopsy.

We present the case of a patient with undifferentiated pleomorphic sarcoma of the lung presenting with symptomatic right-heart failure secondary to cardiac metastasis. The purpose of this report is to present this unusual case.

\section{Case presentation}

A 59-year-old Chinese woman was referred to our hospital 11 months ago because she had had dyspnea for more than 1 month. She had a history of stage IV lung cancer that was located in the left upper lobe. Transthoracic echocardiography revealed no right ventricular tumor at the time the original left lung cancer was diagnosed (Figure 1). The patient was diagnosed by fiberoptic bronchoscopic biopsy. Immunohistochemical analysis demonstrated that the tumor cells were strongly positive for vimentin and CD68, and moderately positive for $\mathrm{Ki}-67(+)$. Focal staining for actin and desmin was also observed, but S-100, CD34(-), CK(pan), CAM5.2 and epithelial membrane antigen (EMA) were all negative. Histopathological and immunohistochemical examinations confirmed a diagnosis of undifferentiated pleomorphic sarcoma 4 years ago. She had successfully undergone multiple sessions of radiotherapy and chemotherapy, and no recurrence was observed during follow-up.

On a physical examination, her blood pressure was $110 / 70 \mathrm{mmHg}$, and her pulse rate was 110 beats per minute and regular. A cardiac examination revealed a right ventricular impulse; a systolic thrill was palpable at the

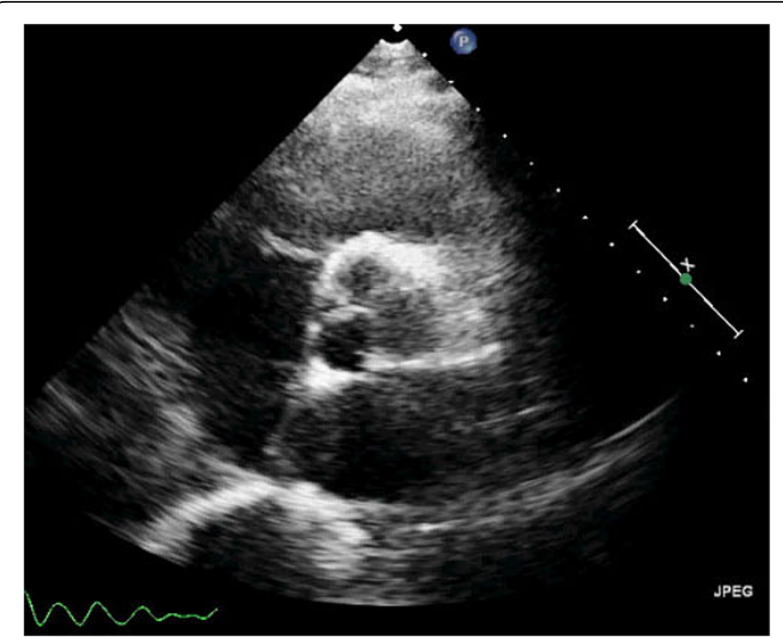

Figure 1 Transthoracic echocardiographic scan (parasternal short-axis view of aortic root) $\mathbf{4}$ years ago. No tumor was revealed in the right ventricle. left sternal border, and a grade III/IV systolic ejection murmur was audible in the same region. On auscultation, her chest was clear.

Contrast-enhanced computed tomography (CT) showed a large mass measuring $5 \mathrm{~cm} \times 6 \mathrm{~cm}$, filling defects in the main pulmonary artery and right ventricle and hydropericardium (Figure 2). No abnormal findings were seen in the lung. Transthoracic echocardiography revealed enlargement of the right ventricle, hydropericardium and a tumor measuring approximately $5 \mathrm{~cm} \times 5 \mathrm{~cm}$ and extending from the right ventricle to the pulmonary valve (Figure 3 ). The liver, spleen, pancreas, and kidneys appeared normal. The patient had no evidence of tumor recurrence in the left lung. Her electrocardiogram showed sinus rhythm with no significant ST-T abnormalities. Serum levels of the tumor markers alphafetoprotein, carcinoembryonic antigen, carcinoma antigen (CA)-125 and CA 19-9 were not elevated.

After considering the extent of the patient's disease, we deemed that an operation was the best alternative. The tumor in the right ventricle and main pulmonary artery was surgically resected; it measured $6 \mathrm{~cm} \times 5 \mathrm{~cm}$ (Figure 4). The postoperative course was uneventful, and she was discharged on day 13 after the surgery. Her dyspnea was significantly relieved postoperatively. Histopathological examination of the resected specimen indicated a diagnosis of undifferentiated sarcoma (Figure 5). Immunohistochemical analysis demonstrated that the tumor cells were strongly positive for vimentin and CD68, and moderately positive for $\mathrm{Ki}-67(+)$. Focal staining for actin and desmin was also observed, but S-100, CD34(-), CK(pan), CAM5.2 and EMA were all negative.

When seen at the last follow-up 2 months ago, our patient was in complete remission, with no evidence of tumor recurrence on $\mathrm{CT}$ and echocardiography.

\section{Discussion}

We have described the first case of cardiac metastasis from an undifferentiated pleomorphic sarcoma of the lung. Immunohistochemistry may be useful to distinguish pulmonary undifferentiated pleomorphic sarcomas from non-mesenchymal malignant tumors and to delineate the level of differentiation of the sarcomas [5]. The limited data available on undifferentiated pleomorphic sarcomas indicate that this neoplasm has an aggressive clinical course, and a high incidence of recurrence and metastasis. Surgical resection is the primary therapeutic modality of this sarcoma. If the tumor is at an advanced stage, combination chemotherapy or radiotherapy may be used as a palliative approach. The overall 5-year survival of patients with undifferentiated pleomorphic sarcoma is approximately 50\% [6]. Our patient was diagnosed with a stage IV, undifferentiated pleomorphic sarcoma of the lung and was treated with multiple cycles of chemotherapy and radiotherapy 4 years ago. This 


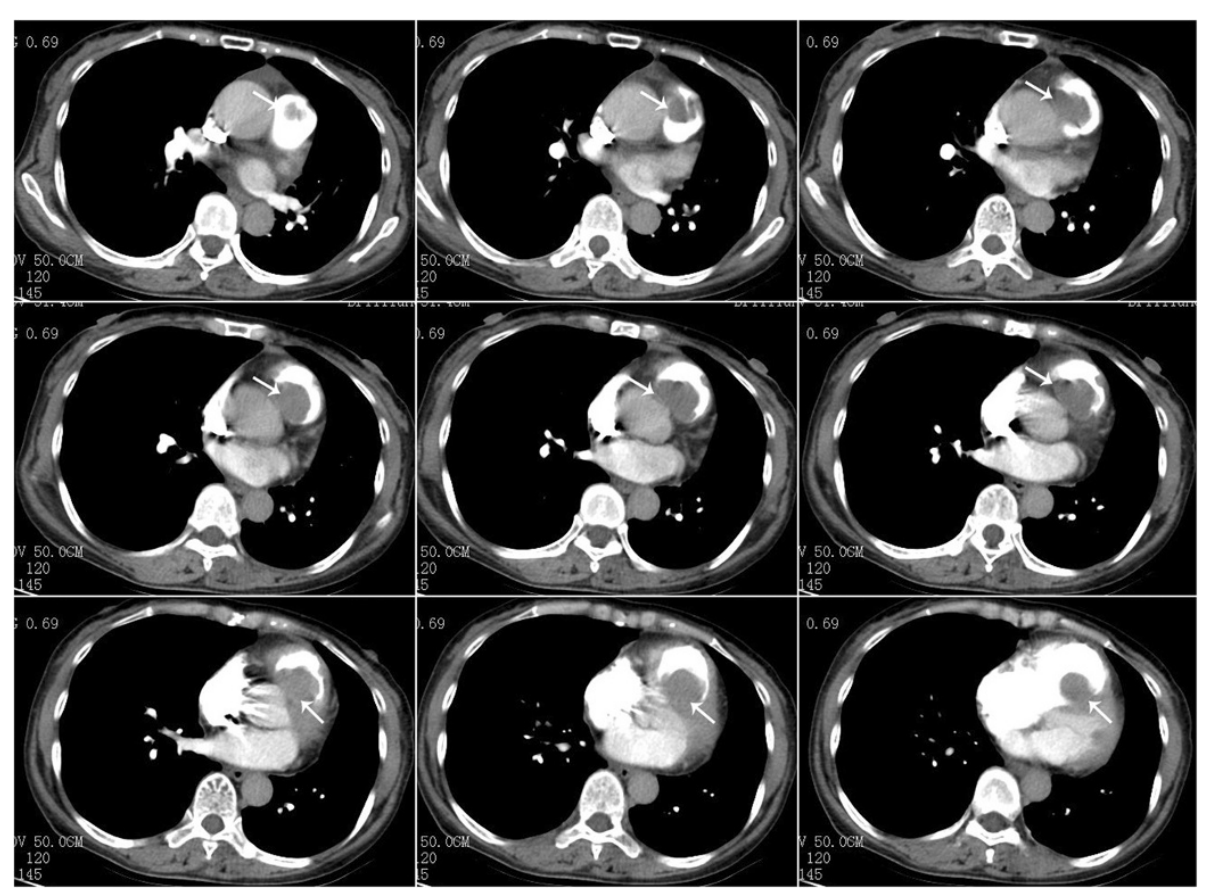

Figure 2 Preoperative, contrast-enhanced, computed tomographic scan of the chest. A large mass measuring $5 \mathrm{~cm} \times 6 \mathrm{~cm}$, filling defects in the main pulmonary artery and right ventricle and hydropericardium can be seen (arrows).

treatment proved efficacious in our patient, and local tumor recurrence did not occur.

Cardiac involvement is not uncommon in lung cancer, and is detected in approximately $25 \%$ of autopsy cases $[7,8]$. In a study by Tamura et al., cardiac involvement was found at autopsy in 23 of 78 patients with lung cancer [9]. Despite the significant mortality and morbidity

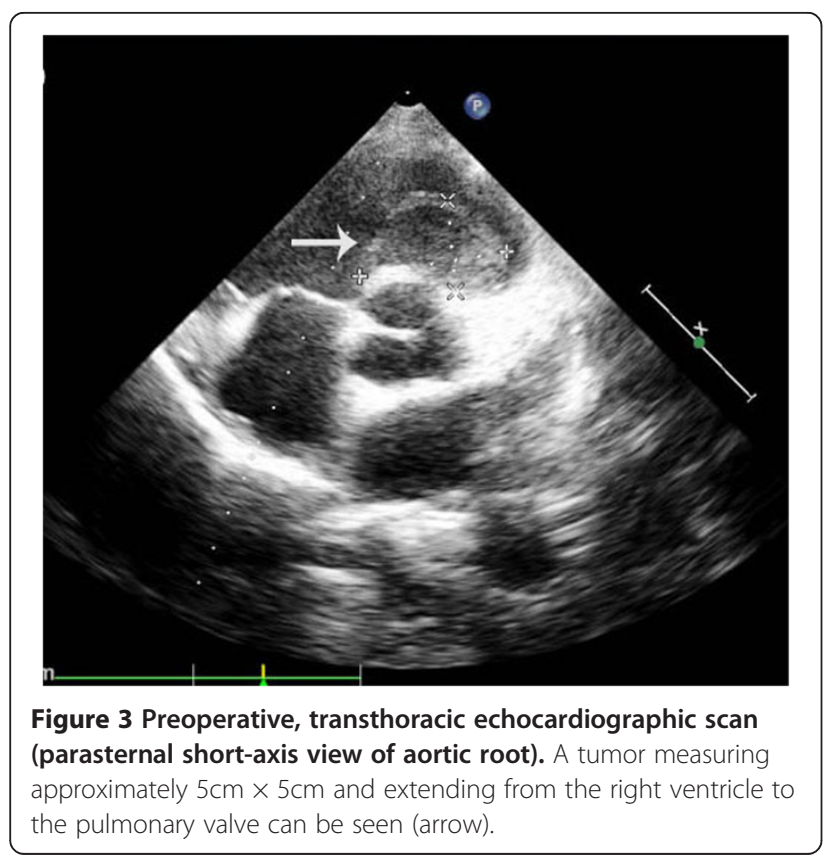

with which it is associated, cardiac metastasis is usually diagnosed during autopsy. Most cardiac metastases are asymptomatic [10]. Only $10 \%$ of patients with cardiac metastasis have symptoms, which are typically related to cardiac function. The clinical presentation varies

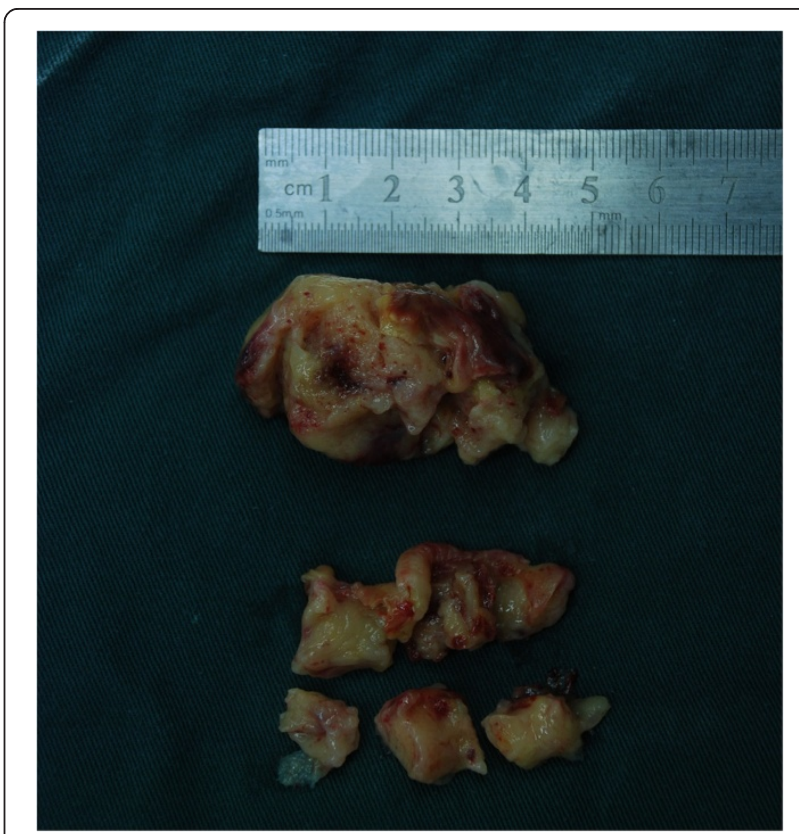

Figure $\mathbf{4}$ Intraoperative specimen. The partially resected tumor obstructed the right ventricular and main pulmonary artery. 


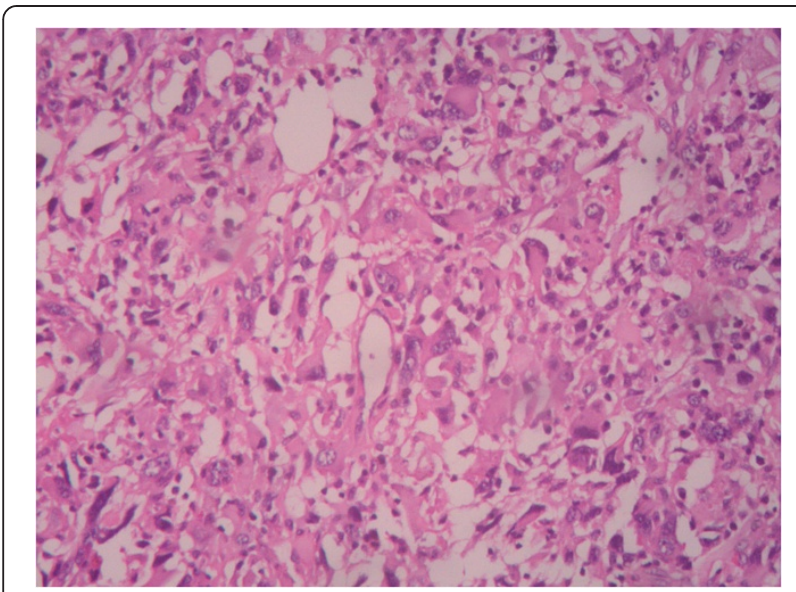

Figure 5 Photomicrograph of resected specimen.

Histopathological examination of the specimen shows that the tumor is an undifferentiated pleomorphic sarcoma (hematoxylin and eosin stain; original magnification, $\times 100$ ).

depending upon the location and extent of myocardial involvement. The small number of reported cases of symptomatic cardiac metastasis attests to the rarity of metastatic lesions that are large enough to cause right ventricular outflow tract obstruction. Our patient presented with dyspnea because of right ventricular outflow tract obstruction. The main differences between primary pulmonary artery sarcoma and metastatic lung disease in the same area is that metastatic lung disease has a history of lung cancer and histopathological and immunohistochemical examinations should be done accordingly with the primary lung cancer. Histopathological and immunohistochemical examinations of the surgical specimen in our patient clearly showed that the cardiac tumor was a metastasis from the lung cancer. Although such cardiac metastases are not uncommon at autopsy, this is the first reported case of a cardiac metastasis from an undifferentiated pleomorphic sarcoma of the lung that was diagnosed in a living patient and was successfully treated with surgery.

Since cardiac metastases are often incurable, surgical resection is not an optimal option for patients with metastatic involvement of the heart. Systemic chemotherapy is usually the most beneficial treatment. Nevertheless, metastatic cardiac involvement poses other problems. Resection of malignant sarcomas is usually only palliative. In cases where the metastasis results in cardiac tamponade or valve obstruction, palliative surgery is often used to relieve symptoms if the primary tumor has been resected in toto, and the patient appears to have a good prognosis. Palliative radiotherapy to the heart is rarely helpful or indicated to relieve symptoms.

In patients with unresectable malignant tumor, palliative surgery may be used, but without great expectation of successful results. Bakaeen et al. [11] reported that the median survival time of patients with malignant cardiac tumors was 9.6 months. Burke et al. [12] reported that the average and median survival in 75 patients with primary malignant cardiac sarcoma was 11 months and 6 months, respectively.

Survival depends on the extent of resection of the sarcomas because of their high potential for recurrence and metastasis. Mean survival after surgical excision has been documented to be 9 to 10 months; a significant predictor of long-term mortality is the presence of New York Heart Association Class III and IV symptoms [11]. The role of adjuvant chemotherapy and radiotherapy is unclear. Given the limited data and the lack of randomized trials, complete surgical resection is currently the only factor that influences survival [13]. In our patient, wide local excision of the recurrent mass was performed, without adjuvant chemotherapy and radiation.

Surgical excision was selected not only because she had had a good outcome after undergoing chemotherapy and radiotherapy 4 years ago but also to prevent intracardiac blood flow obstruction and congestive heart failure. Six months after the surgery, she had recovered well, with no evidence of tumor recurrence on CT and echocardiography.

\section{Conclusions}

Cardiac metastasis from lung cancer is rare and is mainly found at autopsy as an incidental, non-symptomatic finding. In patients who have known metastatic neoplasms and who present with cardiac manifestations, whether detected on history taking or physical examination, the clinician should be alert to the possibility of cardiac metastases. We have reported the first case of symptomatic cardiac metastases from an undifferentiated pleomorphic sarcoma of the lung.

In patients with cardiac metastases, the therapeutic alternatives are limited to palliative treatment of symptoms and chemotherapy. In some patients, surgery can be used to relieve symptoms. Our patient underwent surgical resection, and her symptoms improved significantly. However, treatment of all the metastatic cardiac tumors provides only palliation. Mean survival of affected patients is 3 months to 1 year for metastatic cardiac tumors [14]. The patient will be examined every 3 months by echocardiography in the next 2 years.

This case is unique because it is the only reported case of undifferentiated pleomorphic sarcoma of the lung which metastasized to the heart, and in which symptomatic improvement was obtained after surgical resection.

\section{Consent}

Written informed consent was obtained from the patient for the publication of this case report and the accompanying images. A copy of the written consent is available for review by the Editor-in-Chief of this journal. 


\section{Competing interests}

The authors declare that they have no competing interests in the preparation of this article.

\section{Authors' contributions}

GX drafted the manuscript. GX and XS treated the patient. GS and XS participated in the design of the study. All authors read and approved the final manuscript.

Received: 15 January 2013 Accepted: 2 May 2013

Published: 27 June 2013

\section{References}

1. Fletcher $C D$ : The evolving classification of soft tissue tumours: an update based on the new WHO classification. Histopathology 2006, 48:3-12.

2. Fletcher CDM, Unni KK, Mertens F: World Health Organization classification of tumours. Pathology and genetics of tumours of soft tissues and bone. Lyon, France: IARC Press; 2002:120-122.

3. Pinho T, Rodrigues-Pereira P, Araújo V, Oliveira NP, Macedo F, Graça A, Maciel MJ: Cardiac metastasis of melanoma as first manifestation of disease. Rev Port Cardiol 2009, 5:633-639.

4. Chiles C, Woodard P, Gutierrez F, Link K: Metastatic involvement of the heart and pericardium: CT and MR Imaging. Radiographics 2001, 21:439-449.

5. Al-Nafussi A: Spindle cell tumours of the breast: practical approach to diagnosis. Histopathology 1999, 35:1-13.

6. Zelger B, Burgdorf WH: Fibrohistiocytic tumors. In Skin Cancer. 1st edition Edited by Nouri K. New York: McGraw-Hill; 2007:205-207.

7. Kadappu KK, Rajaratnam R, Kachwalla H, Nguyen PD: Lung cancer mimicking left atrial mass. Postgrad Med J 2008, 84(993):386-387.

8. Bussani R, De-Giorgio F, Abbate A, Silvestri F: Cardiac metastases. J Clin Pathol 2007, 60(1):27-34.

9. Tamura A, Mastubara O, Yoshimura N, Kasuga T, Akagawa S, Aoki N: Cardiac metastasis of lung cancer - a study of metastatic pathways and clinical manifestations. Cancer 1992, 70(2):437-442.

10. Morais VD, Dalbem F, Borges K, Restelli K: Right ventricular tumor in a patient with melanoma. Arg Bras Cardiol 2008, 91(3):e29-e32. e21-24.

11. Bakaeen FG, Chir B, Reardon MJ, Coselli JS, Miller CC, Howell JF, Lawrie GM Espada R, Ramchandani MK, Noon GP, Weilbaecher DG, DeBakey ME: Surgical outcome in 85 patients with primary cardiac tumors. Am J Surg 2003, 186:641-647.

12. Burke AP, Cowan D, Virmani R: Primary sarcomas of heart. Cancer 1992, 84:387-395.

13. Llombart-Cussac A, Pivot X, Contesso G, Rhor-Alvarado A, Delord JP, Spielmann M, Tursz T, Le Cesne A: Adjuvant chemotherapy for primary cardiac sarcomas: the IGR experience. Br J Cancer 1998, 78:1624-1628.

14. Grebenc ML, Rosado de Christenson ML, Burke AP: Primary cardiac and pericardial neoplasms: radiologic-pathologic correlation. Radiographics 2000, 20:1073-1103.

doi:10.1186/1752-1947-7-165

Cite this article as: Xu et al: An unusual case of metastasis of a pulmonary undifferentiated pleomorphic sarcoma to the right ventricle: a case report. Journal of Medical Case Reports 2013 7:165.

\section{Submit your next manuscript to BioMed Central and take full advantage of:}

- Convenient online submission

- Thorough peer review

- No space constraints or color figure charges

- Immediate publication on acceptance

- Inclusion in PubMed, CAS, Scopus and Google Scholar

- Research which is freely available for redistribution 